\title{
Análise estatística da qualidade de níveis de tensão em sistemas de distribuição de energia elétrica
}

\author{
Guilherme Luís Roehe Vaccaro, ${ }^{\mathrm{a}, *}$, Jonatas Campos Martins ${ }^{\mathrm{b}}$, Thiago Morais Menezes ${ }^{\mathrm{c}}$ \\ a,* guilhermev@unisinos.br, UNISINOS, Brasil \\ bjonatascm@gmail.com, UNISINOS, Brasil \\ cthiagomoraismenezes@gmail.com, UNISINOS, Brasil
}

\begin{abstract}
Resumo
Este trabalho apresenta uma proposta de abordagem estatística para análise da qualidade dos níveis de tensão em sistemas de distribuição de energia elétrica baseada nos princípios estabelecidos pela Resolução n. ${ }^{\circ}$ 505/2001 da Agência Nacional de Energia Elétrica - ANEEL, à qual todas concessionárias de distribuição de energia do país estão submetidas. 0 artigo faz considerações sobre qualidade na distribuição de energia elétrica, os procedimentos vigentes para o controle da qualidade dos níveis de tensão e evidencia a possibilidade de uso de conceitos do controle estatístico do processo e de índices de capacidade e desempenho como elementos alternativos de informação e predição. Um estudo de caso em dois circuitos de distribuição de baixa tensão é analisado de forma comparativa, usando-se primeiramente os procedimentos e critérios estabelecidos pela ANEEL e, em seguida, a abordagem proposta pelos autores. 0 artigo finaliza com uma análise das potenciais contribuições do procedimento estatístico proposto aos procedimentos vigentes.
\end{abstract}

Palavras-chave

Distribuição de energia elétrica. Controle do nível de tensão. Qualidade. Controle estatístico do processo. Autocorrelação.

\section{Introdução}

A energia é um ingrediente essencial do desenvolvimento socioeconômico e do crescimento econômico (GOLDEMBERG, 1998). Hoje em dia, a evolução, o crescimento e o desenvolvimento de um país podem ser diretamente relacionados à disponibilidade energética. A disponibilidade de energia implica transformações que se revertem em melhorias nas dimensões econômicas, sociais, ambientais e institucionais do desenvolvimento da economia nacional (SCHAEFFER et al., 2003). Desde o descobrimento da energia elétrica e sua ampla utilização no final do século XIX, o homem vem se tornando cada vez mais dependente desse insumo (ITO, 2003). No Brasil, as iniciativas governamentais no setor de energia elétrica foram fundamentais para o crescimento da disponibilidade desse insumo, com a viabilização do surgimento das companhias de eletricidade, construções de usinas elétricas e criação de órgãos públicos vinculados ao setor energético, com fins de planejamento e regulamentação.

Segundo o modelo brasileiro de concessões e comercialização de energia, as empresas dividem-se em três segmentos: geração, transmissão e distribuição. As empresas de distribuição prestam serviço em regiões específicas, denominadas áreas de concessão, tendo a missão de atender às comunidades residenciais, comerciais e industriais quanto ao fornecimento de energia elétrica. Para participar da distribuição de energia, as empresas devem concorrer em processos licitatórios abertos, conforme a legislação vigente (BRASIL, 2008). Como parte do processo licitatório, um contrato de concessão de distribuição de energia elétrica é firmado entre a empresa distribuidora vencedora na referida área de concessão e o governo, por meio de seu representante, a ANEEL - Agência Nacional de Energia Elétrica. 
Os contratos de concessão definem padrões para a manutenção da qualidade e do aprimoramento dos serviços prestados pelas concessionárias de energia elétrica, fornecendo como referência parâmetros para a distribuição de energia e de atendimento a prazos para realização das atividades, denominados índices de qualidade e frequência de fornecimento (BRASIL, 2008). Esses parâmetros particulares estão associados à regulamentação oficial do setor e suas atualizações, durante todo o período de concessão. Entre esses parâmetros encontra-se a especificação técnica do nível de tensão fornecido para os consumidores, o qual é garantia de atendimento das especificações técnicas de funcionamento de equipamentos elétricos e eletrônicos.

Dada sua importância, a regulação da qualidade da energia elétrica é foco de ocupação da ANEEL. Um dos principais documentos regulatórios para o setor de distribuição de energia elétrica é a Resolução n. 505 da ANEEL, de 26 de novembro de 2001 (R505/2001), a qual estabelece as disposições relativas à conformidade dos níveis de tensão de energia elétrica em regime permanente e especifica a metodologia para medição de níveis de tensão (BRASIL, 2001). Segundo a metodologia estabelecida, a avaliação requer investimentos em sensores e pessoal, muitas vezes considerados elevados pelas concessionárias, do ponto de vista de seu custo. No entanto, segundo Jannuzi (2007), é fundamental respeitar o direito de recebimento de energia elétrica com qualidade compatível com os requisitos de utilização (em consonância com os limites adequados dos indicadores fixados em resolução específica da ANEEL), bem como estabelecer a compensação efetiva e automática por tensão inadequada a unidades consumidoras faturadas com a mesma tarifa de energia elétrica.

Em função do contexto apresentado, este artigo tem como objetivo discutir a utilização de uma abordagem estatística alternativa baseada em conceitos do controle estatístico de processo (MONTGOMERY, 2004; LIN; CHOU, 2004) para análise da qualidade do nível de tensão de energia elétrica em regime permanente. Metodologicamente, pode ser descrito como um estudo quantitativo com características de estudo de caso exploratório (YIN, 2005; GlL, 1996). 0 artigo apresenta uma análise quantitativa dos níveis de tensão elétrica de dois circuitos de distribuição. A abordagem proposta é comparada ao procedimento estabelecido pela ANEEL, conforme a Resolução n. ${ }^{\circ}$ 505/2001, no que tange à forma de tratamento dos dados.

0 restante do artigo está estruturado em: considerações sobre qualidade da distribuição de energia elétrica e política de controle de níveis de tensão; referencial teórico sobre controle estatístico do processo e índices de capacidade e desempenho do processo; metodologia; análise do estudo, implicações e constatações; conclusões e sugestões. Como resultado, espera-se contribuir para a qualificação do serviço prestado pelas concessionárias de energia, focalizando o potencial de redução de custos de avaliação dos níveis de tensão sem redução dos padrões de qualidade estabelecidos.

\section{Qualidade da distribuição de energia elétrica}

A qualidade do serviço prestado pelas empresas do setor elétrico de distribuição de energia pode ser avaliada nos seguintes aspectos: a continuidade do fornecimento; a qualidade do atendimento ao consumidor; e a qualidade do produto energia elétrica (BELISÁRIO; BAHIENSE; OLIVEIRA, 2003). Esses aspectos são pontos básicos para a definição dos diversos critérios de localização e arranjo das subestações; a localização das equipes de serviços; os critérios de escolha dos materiais e equipamentos de controle e proteção; a regulação de tensão; e a configuração da rede de distribuição.

Segundo Paniago (2006), cada um dos três aspectos é assim entendido: a continuidade do fornecimento corresponde à disponibilidade da energia elétrica ao consumidor e é caracterizada por dois parâmetros - duração e frequência das interrupções; a qualidade do atendimento abrange a relação comercial existente entre a concessionária e o consumidor, considerando-se a cortesia, o tempo de atendimento às solicitações de serviços, o grau de presteza, o respeito aos direitos do consumidor, entre outros; a qualidade do produto está relacionada com os fenômenos associados à forma de onda de tensão, tais como flutuações de tensão, distorções harmônicas, variações momentâneas de tensão, entre outros.

Segundo a ANEEL (BRASIL, 2008), os parâmetros que caracterizam a qualidade da energia elétrica fornecida, tanto em regime permanente como transitório, são:

- Tensão em regime permanente: tensão no estado estacionário, para a qual são estabelecidos os limites adequados, precários e críticos, os indicadores de qualidade, os critérios de medição e registro, os prazos para regularização e de compensação ao consumidor, caso os limites para o atendimento adequado não sejam obedecidos;

- Fator de potência: a razão entre a energia elétrica ativa e a raiz quadrada da soma dos quadrados das energias elétrica ativa e reativa, consumidas num mesmo período de tempo, sendo a energia 
elétrica reativa a que circula continuamente entre os diversos campos elétricos e magnéticos de um sistema de corrente alternada, ou por efeito de chaveamento, sem produzir trabalho, expressa por quilovolt-ampére-reativo-hora (kVArh);

- Distorções harmônicas: fenômenos associados com deformações nas formas de onda das tensões e correntes em relação à onda senoidal da frequência fundamental;

- Desequilíbrio de tensão: o fenômeno associado a alterações dos padrões trifásicos do sistema de distribuiç̧ão;

- Flutuação de tensão: uma variação aleatória, repetitiva ou esporádica do valor eficaz da tensão; e

- Variações de tensão de curta duração: desvios significativos no valor eficaz da tensão em curtos intervalos de tempo.

Segundo Jannuzi (2007), fundamentalmente, a qualidade da energia elétrica está diretamente relacionada à qualidade da tensão. Segundo o autor, nos sistemas elétricos pode-se controlar somente a tensão, não sendo possível exercer qualquer controle sobre a corrente elétrica com vistas a melhorar a qualidade da energia elétrica. Assim, a perda na qualidade da energia elétrica é qualquer anomalia na forma de onda da tensão elétrica, na corrente ou na frequência que resulte em falha ou operação inadequada de um equipamento elétrico. Do ponto de vista do consumidor, é possível definir qualidade da energia elétrica como sendo o recebimento de energia elétrica com regime de tensão em conformidade com parâmetros estabelecidos em regulamentação específica do órgão regulador e compatível com os requisitos técnicos necessários à operação adequada do equipamento eletroeletrônico, de forma a garantir a vida útil do mesmo, segundo especificações técnicas de fabricantes idôneos (JANNUZl, 2007).

\subsection{Contrato de concessão e níveis de tensão de distribuição}

As redes de distribuição de energia elétrica no Brasil possuem características que devem ser mencionadas a fim de facilitar a compreensão do leitor quanto ao presente trabalho. 0 contrato de concessão é o instrumento que disciplina os direitos e as obrigações da concessionária encarregada da prestação de um serviço público. Dentre outras cláusulas essenciais que devem constar num contrato de concessão, a Lei no 8987 , de 1995, impõe em seu inciso $\mathrm{lll}$, art. 23, a necessidade do estabelecimento de meios precisos para aferição da qualidade do serviço prestado. Esses meios são os critérios, os indicadores, as fórmulas e os parâmetros com base nos quais será avaliada a qualidade do serviço (BRASIL, 2009; BLANCHET, 1999).

Conforme Méffe et al. (2002), as redes de distribuição do país são divididas em média e baixa tensão. As redes de média tensão apresentam, em sua maioria, tensões entre 13,8 kV e 34,5 kV, possuindo configurações radiais. São redes trifásicas no tronco, podendo ter ramais bifásicos e monofásicos (este último comumente utilizado em áreas rurais). Seu comprimento varia desde dezenas até centenas de quilômetros. Tais redes alimentam transformadores de distribuição, cujo objetivo é reduzir a tensão para níveis de $220 \mathrm{~V}$ entre fases e $127 \mathrm{~V}$ entre fase e neutro. Cada transformador de distribuição alimenta uma rede de baixa tensão, composta por um cabo neutro e um ou mais cabos de fase (até 3 fases), cujo comprimento varia desde algumas dezenas até centenas de metros.

Das redes de baixa tensão partem ramais de serviço até o ponto de entrega aos consumidores de baixa tensão. Esse ponto de entrega, individual por consumidor, é o ponto em que o nível de tensão deve ser medido, tanto em caso de reclamação por parte do cliente sobre as condições de fornecimento quanto para atendimento do programa periódico de medições feito pela concessionária de distribuição de energia elétrica (de acordo com as diretivas dadas pelo órgão regulador). Os níveis de tensão efetivamente medidos (Tensão de Leitura - TL) são comparados aos limites de tensão exigidos para garantir a qualidade do fornecimento quanto à conformidade de tensão. Esses limites são baseados nos valores de Tensão Nominal (TN), conforme apresenta o Quadro 1.

Assim, para cada valor de tensão nominal há uma faixa de tensão de leitura considerada adequada, com intervalos superiores e inferiores considerados precários e críticos. Por exemplo, para uma ligação trifásica com tensão nominal de $127 \mathrm{~V}$, as faixas ficariam apresentadas conforme a Figura 1.

Como afirmado anteriormente, regular e fiscalizar a qualidade dos serviços prestados aos consumidores são atribuições da ANEEL. Por meio da R505/2001, a ANEEL revisou e atualizou as disposições relacionadas à conformidade dos níveis de tensão da distribuição de energia elétrica no país. Essa resolução apresenta um caráter rigoroso em relação à observância dos indicadores de conformidade dos níveis de tensão de energia elétrica em regime permanente, estabelecidos para serem atendidos por parte das concessionárias. A esses níveis denomina-se Tensão de Atendimento (TA), a qual é formalmente definida como o valor eficaz de tensão no ponto de entrega ou de conexão, obtido por meio de medição, podendo ser classificada em adequada, precária ou crítica, de acordo 
Quadro 1. Extrato da tabela 4 da Resolução n. ${ }^{\circ}$ 505/2001 da ANEEL, apresentando as tensões nominais padronizadas em valores absolutos.

\begin{tabular}{|c|c|c|c|c|}
\hline \multicolumn{2}{|c|}{ Tensões nominais padronizadas } \\
\hline \multicolumn{2}{|c|}{ Tensão nominal (TN) } & $\begin{array}{c}\text { Faixa de valores adequados } \\
\text { das tensões de leitura (TL) } \\
\text { em relação à TN }\end{array}$ & $\begin{array}{c}\text { Faixa de valores precários das } \\
\text { tensões de leitura (TL) } \\
\text { em relação à TN }\end{array}$ & $\begin{array}{c}\text { Faixa de valores críticos das } \\
\text { tensões de leitura (TL) } \\
\text { em relação à TN }\end{array}$ \\
\cline { 1 - 2 } Ligação & Volts & \multirow{2}{*}{$0,91 \mathrm{TN} \leq \mathrm{TL} \leq 1,04 \mathrm{TN}$} & $\begin{array}{c}0,86 \mathrm{TN} \leq \mathrm{TL}<0,91 \mathrm{TN} \text { ou } \\
1,04 \mathrm{TN}<\mathrm{TL} \leq 1,06 \mathrm{TN}\end{array}$ & $\begin{array}{c}\mathrm{TL}<0,86 \mathrm{TN} \text { ou } \\
\mathrm{TL}>1,06 \mathrm{TN}\end{array}$ \\
\cline { 1 - 2 } Trifásica & $220 / 127$ & $380 / 220$ & \\
\cline { 2 - 2 } Monofásica & $254 / 127$ & & &
\end{tabular}

Fonte: ANEEL (BRASIL, 2008).

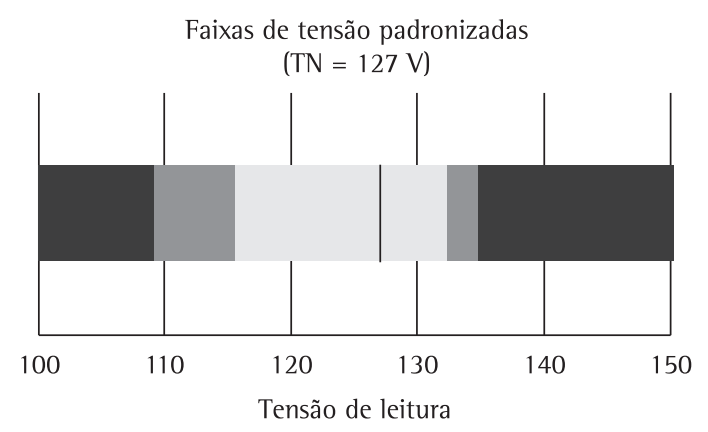

Crítico $\square$ Precário $\square$ Adequado $\square$ Nominal

Figura 1. Faixas de tensão padronizadas para ligação trifásica de $127 \mathrm{~V}$.

com a leitura efetuada, expressa em volts ou quilovolts (BRASIL, 2001). Ou seja, a TA refere-se à classificação da tensão no ponto de conexão ou entrega do serviço da distribuidora. Com isso a ANEEL visou estimular a melhoria contínua da qualidade dos serviços prestados aos consumidores (BRASIL, 2008).

Ao dedicar-se ao tratamento dos níveis de tensão efetivamente medidos nas redes das concessionárias, a ANEEL refinou a discussão e os requisitos sobre a qualidade da energia elétrica entregue ao consumidor. A proposta da ANEEL especifica:

- A abordagem metodológica para medição de nível de tensão;

- As características mínimas dos equipamentos e dos softwares de processamento de sinais;

- Os prazos para regularização dos níveis; e

- As multas a serem aplicadas em caso de não cumprimento das especificações.

De acordo com a R505/2001, a ANEEL estabelece como um direito do consumidor solicitar à concessionária a medição do nível da tensão de atendimento sempre que acreditar que este não está dentro dos limites legalmente estabelecidos, devendo esta lhe informar, no prazo de até 48 horas, o valor que será cobrado pelo serviço (na hipótese da constatação da improcedência da reclamação), bem como a data e hora em que será instalado o equipamento para acompanhamento da medição pelo solicitante. 0 relatório conclusivo da medição deve ser apresentado, por escrito, ao consumidor solicitante em até 30 dias a partir da formalização de sua reclamação.

\subsection{Procedimentos e indicadores de qualidade dos níveis de tensão de distribuição}

A ANEEL estabelece que a concessionária deve ter um programa periódico trimestral de medições, no qual é selecionada uma amostra de unidades consumidoras para apuração dos indicadores de conformidade de tensão (BRASIL, 2008). As medições devem ter duração mínima de 168 horas, com intervalos de integração de 10 minutos, a partir de leituras com janelas fixas e consecutivas de 12 a 15 ciclos, totalizando 1.008 registros válidos. As medições devem ser feitas entre fases e entre fases e o neutro. Os indicadores a serem apurados são:

- DRP - Duração relativa da transgressão de tensão precária; e

- DRC - Duração relativa da transgressão de tensão crítica.

0 DRP expressa o percentual de leituras realizadas nas faixas precárias de tensão em relação ao total de leituras realizadas no período de medição. De forma análoga, o DRC expressa o percentual de leituras realizadas nas faixas críticas de tensão como um percentual do total de leituras realizadas no período de medição. 0 cálculo dos índices é realizado da seguinte forma:

- DRP é a razão entre as medições na faixa precário e a quantidade total de medições;

- DRC é a razão entre as medições na faixa crítico e a quantidade total de medições. 
0 limite máximo a ser observado para o DRP foi estabelecido em 7\% para o ano de 2004, sendo reduzido de um valor absoluto de $1 \%$ a cada ano, no período de 2005 a 2007. A partir de 2008 passou a ter o valor fixo de 3\%. Da mesma forma, o limite máximo a ser observado para o DRC foi estabelecido em 1,1\% para o ano de 2004, sendo este reduzido de um valor absoluto de $0,2 \%$ a cada ano, no período de 2005 a 2007. A partir de 2008 passou a ter o valor fixo de 0,5\% (BRASIL, 2008).

0 procedimento vigente foca-se em estabelecer regras para subsidiar os agentes e consumidores do sistema elétrico nacional, disciplinando condições, responsabilidades e penalidades relativas à conexão, planejamento da expansão, operação e medição da energia elétrica, sistematizando a troca de informações entre as partes, além de estabelecer critérios e indicadores de qualidade. Em caso de ocorrência de irregularidade, a adequação do nível de tensão deve ser comprovada por nova medição, obedecendo ao mesmo período de observação, e o resultado final comunicado, por escrito, ao consumidor que efetuou a reclamação, no prazo de até 30 dias após o término da nova medição.

\section{Qualidade, variabilidade e controle estatístico do processo}

A variabilidade é inerente a qualquer tipo de processo. Isso significa que todo processo, mesmo operando em condições desejadas de serviço, apresenta variabilidade nos itens que produz. Em processos produtivos, por exemplo, as medidas de duas peças podem diferir por causa do desgaste de uma ferramenta, natureza do material, ou mesmo da temperatura do equipamento de processamento. Tempos de atendimento em serviços também são exemplos de operações que apresentam variabilidade. A variabilidade sempre existirá e a sua eliminação completa não é o objetivo principal de uma organização. No entanto, deve haver uma preocupação em identificar as causas da variabilidade, com o objetivo de reduzi-la, facilitando os processos de gestão e minimizando os riscos de não conformidades (MONTGOMERY; RUNGER, 2003).

A conformidade de um produto ou processo está diretamente relacionada às especificações sob as quais esse produto ou processo está submetido (FEIGENBAUM, 1983; MONTGOMERY, 2004). Ela constitui uma das visões possíveis de qualidade, quando esta é entendida como a capacidade de um produto ou serviço em atender às expectativas de um indivíduo ou organização com relação a um padrão (tangível ou intangível) de satisfação (DEMING 2000). Esse aspecto da qualidade é também referido como a adequação ao uso e às condições de uso (JURAN, 1992; AKAO; MIZUNO, 1994). Como conceito integrador, Montgomery (2004) define qualidade como o inverso da variabilidade, indicando também a necessidade de conformidade com especificações.

Do ponto de vista das relações entre cliente e consumidor, contudo, o conceito acordado de qualidade deve ser claro e objetivo. Isso significa que a empresa fornecedora deve estar ciente das necessidades de seus clientes e, em função destas, definir os requisitos de qualidade do produto. 0 processo deve ser acompanhado e seu desempenho deve ser avaliado por procedimentos focados nas características de qualidade (DEUS; VACCARO, 2008).

0 Controle Estatístico do Processo (CEP) consiste na aplicação de um conjunto de técnicas estatísticas para determinar se o resultado do processo segue as especificações estabelecidas para um produto ou serviço. Ele fundamenta-se em três princípios da probabilidade e estatística, a saber: o teorema do limite central, a estratificação das causas da variabilidade entre comuns e especiais, e o conceito de independência entre observações (MONTGOMERY, 2004; LIN; CHOU, 2004).

0 teorema do limite central garante que a distribuição da média dos dados de uma população qualquer tenda a seguir um padrão normal. A estratificação entre causas comuns e especiais permite focalização em ações consideradas significantes para a melhoria do processo. Tipicamente, as causas comuns são as pequenas causas que atuam de forma aleatória e inevitável sobre o processo e geralmente requerem intervenções sobre todo o sistema para que sejam eliminadas. Causas especiais, ou assinaláveis, seguem padrões sistemáticos de ocorrência, tendo efeito expressivo sobre o sistema. Podem ser eliminadas por ações focadas, atuando sobre um recurso, equipamento ou condição operacional, por exemplo.

Montgomery (2004) cita alguns objetivos do CEP que justificam a sua utilização:

- Permite predizer até que ponto o processo manterá as tolerâncias estabelecidas;

- Auxilia os elaboradores/planejadores de um produto na seleção de modificações de um processo;

- Auxilia a estabelecer intervalos entre amostras para monitoramento de um processo;

- Permite especificar exigências de desempenho para um equipamento novo;

- Permite planejar a sequência de processos de produção quando há um efeito interativo de processo sobre as tolerâncias;

- Permite reduzir a variabilidade em um processo. 
Uma das ferramentas mais conhecidas do CEP são as cartas de controle estatístico. Originariamente desenvolvidas por Walter Shewhart na década de 20, e formalmente apresentadas em 1931 (SHEWHART, 1931), estabelecem suposições-padrão sobre as quais se pode realizar o monitoramento de um processo. As premissas tipicamente estabelecidas são que os dados gerados pelo processo, quando sob controle, são distribuídos normal e independentemente com média $\mu$ e desvio padrão $\sigma$. Tanto $\mu$ como $\sigma$ são considerados fixos e desconhecidos. Uma condição fora de controle é a mudança ou deslocamento de $\mu$ ou $\sigma$ (ou ambos) para algum valor sensivelmente diferente. Quando essas suposições são satisfeitas, os gráficos de controle permitem estabelecer conclusões sobre o estado de controle estatístico do processo.

Mesmo em situações em que a condição de normalidade é violada em grau moderado, os gráficos de controle ainda funcionam razoavelmente bem (MONTGOMERY, 2004). A suposição mais importante relativa aos gráficos de controle é a de independência das observações (MONTGOMERY, 2004), porque os gráficos de controle convencionais não funcionam bem se a característica da qualidade apresenta níveis, ainda que baixos, de correlação ao longo do tempo. Especificamente, esses gráficos de controle darão resultados enganosos sob a forma de demasiados alarmes falsos se os dados forem correlacionados.

Em estatística, autocorrelação de uma medida informa o grau de dependência entre valores sucessivos de uma série aleatória. Em um processo autocorrelacionado positivamente, por exemplo, um valor acima da média tende a ser seguido por outro valor acima da média, enquanto um valor abaixo da média é, usualmente, seguido por outro valor do mesmo tipo. Isso produz uma série de dados que tem uma tendência a se mover em "sequências" moderadamente longas em cada um dos lados da média. Como consequência, corre-se o risco de subestimar a variabilidade intrínseca ao processo, em função da proximidade entre observações sucessivas.

Quando possível, uma amostragem menos frequente pode interromper a autocorrelação nos dados do processo (MONTGOMERY, 2004). Ou seja, uma abordagem para tratar a autocorrelação consiste simplesmente em extrair amostras do fluxo de dados do processo com menos frequência, mas ainda sob as mesmas condições operacionais. Outra possibilidade é a utilização de médias de lotes, de forma similar à adotada para a coleta de dados de controle de processo. Uma terceira opção é tentar modelar diretamente a estrutura de correlação da série de dados, usando modelos de séries temporais autorregressivos, como os de primeira ordem, ou AR(1) (CLARO; COSTA; MACHADO, 2007).

\subsection{Indices de capacidade do processo $\left(C_{p}\right.$ e $\left.C_{p k}\right)$}

A análise de capacidade de um processo consiste em uma abordagem metodológica baseada na probabilidade e estatística, para mensurar a adequação do comportamento de uma ou mais características de interesse quanto a padrões previamente estabelecidos. Segundo Torminato (2004), a análise de capacidade é parte importante do CEP, pois permite ao especialista determinar a habilidade do processo em satisfazer as especificações e os limites de tolerância do processo, além de diagnosticar o estado de controle dos processos de produção, verificando se são ou não capazes de satisfazer as solicitações dos clientes.

Os indices de capacidade do processo comumente utilizados são (MONTGOMERY, 2004; TORMINATO, 2004):

Índice de capacidade $\left(C_{p}\right)$ : Mede a capacidade potencial do processo, ou seja, sua capacidade de atender as especificações se o mesmo estiver ajustado. É definido como o intervalo de tolerância dividido pela amplitude do processo, ou seja, 6 vezes o desvio padrão estimado, considerando a ausência de causas especiais, como na Equação 1:

$C p=\frac{L S E-L I E}{6 \sigma}$

onde:

LSE e LIE representam respectivamente o limite superior e inferior de especificação e $\sigma$ é o desvio padrão do processo.

0 índice $C_{p}$ não avalia a centralização do processo, isto é, a relação entre a média estimada do processo, $\bar{X}$, com os limites de especificação (KAMINSKY; DOVICH; BURKE, 1998; MONTGOMERY, 2004; GONÇALEZ; WERNER, 2009). Assim o $C_{p}$ não representa a porcentagem fora de especificação, mas simplesmente um indice de capacidade nominal do processo. Numericamente, se $C_{p}$ for igual ou superior a 2,0, o processo é considerado capaz, indicando que a faixa de especificação é duas vezes mais ampla que sua dispersão intrínseca. Se $C_{p}$ for igual a 1,0, o processo é considerado marginalmente capaz, dado que a faixa de especificação é a mesma que sua faixa de dispersão. Por outro lado, se $C_{p}<1,0$, o processo não pode ser considerado capaz, dado que a faixa de especificação é menor que sua faixa de dispersão natural. Segundo Montgomery (2004), valores entre 1 e 2 são considerados aceitáveis. Num processo já existente, o valor mínimo recomendado é de 1,33 e, para processos novos, o valor sugerido é de 1,5 . 
Índice de capabilidade $\left(\mathrm{C}_{\mathrm{pk}}\right)$ : mede a capacidade efetiva do processo. Considera simultaneamente se o processo possui uma dada dispersão e se a média atende as especificações levando em conta a centralização do processo e é definido como o mínimo entre o limite superior e o inferior de capabilidade, conforme a Equação 2:

$$
C_{p k}=\min \left(C_{p u}, C_{p l)}=\min \left(\frac{L S E-\mu}{3 \sigma}, \frac{L I E-\mu}{3 \sigma}\right)\right.
$$

onde $\mu$ representa a média do processo e os demais elementos são apresentados como na Equação 1.

0 índice $C_{p k}$ mede quantos desvios padrões estão situados no intervalo entre a média do processo e o limite especificado mais próximo. Quanto maior o $C_{p k}$, menor a dispersão do processo ou menor o afastamento da média do processo em relação ao alvo. Numericamente, segundo a ASQC (AMERICAN..., 1986), o valor mínimo recomendado para o $C_{p k}$ é de 1,33. Ainda, se $C_{p k}$ for igual a 2,0, o processo é capaz e está oscilando em apenas 50\% da especificação; se $C_{p k}$ for igual a 1,0 , a capacidade do processo é marginal; e se $\mathrm{C}_{\mathrm{pk}}$ for menor que 1,0 , o processo não pode ser considerado capaz. $0 C_{p k}$ mede a capacidade que um processo possui em produzir resultados efetivamente aceitáveis, ou seja, variando dentro dos limites especificados de projeto. Caso o conjunto de dados siga distribuições diferentes da distribuição normal, outras expressões devem ser usadas, sendo, neste caso, necessário ajustar previamente um modelo probabilístico adequado e usar índices como o $\mathrm{C}_{\mathrm{PM}}, \mathrm{o}_{\mathrm{NPJ}}$ e o $\mathrm{C}_{\mathrm{Npmk}}$, por exemplo. Caso contrário, corre-se o risco de superestimar o $C_{\mathrm{pk}}$ (MONTGOMERY, 2004). Uma discussão detalhada sobre o tema pode ser encontrada em Gonçalez e Werner (2009).

\section{2. Índices do desempenho potencial de um processo $\left(P_{p}\right.$ e $\left.P_{p k}\right)$}

Segundo Montgomery (2004), a AIAG (Automotive Industry Action Group) recomenda o uso dos índices de capacidade de um processo $C_{p}$ e $C_{p k}$ quando o processo está sob controle, com o desvio padrão estimado por $\sigma=\bar{R} / \mathrm{D}_{2}$, onde R é a amplitude e o coeficiente $D_{2}$ é uma estimativa da média da distribuição $R / \sigma$, a qual é influenciada também pelo tamanho da amostra coletada. Tal procedimento é utilizado em função da maior facilidade, em ambientes produtivos, de se estimar a amplitude de variação de um processo em lugar de seu desvio padrão.

Quando o processo não está sob controle, a AIAG recomenda o uso dos índices do desempenho de um processo $\mathrm{P}_{\mathrm{p}}$ e $\mathrm{P}_{\mathrm{pk}}$. Esses índices analisam se a amostra gerada do processo tem condição de atender as especificações estabelecidas, não sendo adequados para inferências sobre a condição geral do processo. As expressões para esses índices são:

$$
P p=\frac{L S E-L I E}{6 S}
$$

e

$$
P_{p k}=\min \left(\frac{L S E-\bar{X}}{3 S}, \frac{L I E-\bar{X}}{3 S}\right)
$$

onde $\bar{X}$ é uma estimativa de $\mu$; $S$ é o desvio padrão amostral. Também o American National Standard Institute, em sua ANSI Standard Z1 on Processes Capability Analysis, afirma que $\mathrm{P}_{\mathrm{p}}$ e $\mathrm{P}_{\mathrm{pk}}$ devem ser usados quando o processo não está sob controle (MONTGOMERY, 2004).

\subsection{Autocorrelação}

Um dos pressupostos de grande parte dos procedimentos clássicos de análise estatística é o de que os dados utilizados sejam provenientes de variáveis aleatórias independentes e identicamente distribuídas. Nesta situação ideal, dada uma variável aleatória $X$, há garantias da estatística clássica de que a média e a variância de uma amostra de $X$ podem ser calculadas e corretamente representadas pelos estimadores da média e da variância.

0 problema do uso de expedientes de análise estatística sem a devida análise desse pressuposto é que, se a hipótese de independência não for cumprida, gera-se um viés de interpretação, correndo-se o risco de subestimar o efeito da variabilidade do processo. A identificação de situações desta natureza é importante e pode passar despercebida ao usuário menos experiente.

A perda de independência entre observações consecutivas é o que se denomina autocorrelação (MONTGOMERY, 2004). Em dados positivamente autocorrelacionados mudanças tendem a acontecer de modo mais lento. Como consequência, estimativas de variâncias de dados resultantes de simulação serão em geral menores que os valores reais de variância para o sistema. Consequentemente, intervalos de confiança baseados em tais dados tenderão a parecer mais estreitos, levando erroneamente a crer que os resultados da simulação incluem muito menos erros do que na situação real. Assim, sobre dados autocorrelacionados, a cobertura (efetividade) dos intervalos de confiança é, geralmente, pobre (THESEN; TRAVIS, 1990).

Mais precisamente, se $X$ for uma variável aleatória com variância $\sigma^{2}$, a correlação entre $x_{i}$ e $x_{i+j}$ para qualquer observação i será dada por (MONTGOMERY, 2004) (Equação 4): 


$$
\rho_{j}=\frac{\operatorname{Cov}\left(X_{i}, X_{i+j}\right)}{\sigma_{X_{i}} \sigma_{X_{i+j}}}
$$

que pode ser estimada por

$$
\hat{\rho}_{j}=\frac{\sum_{i=1}^{n-j}\left(X_{i}-\bar{X}\right)\left(X_{i+j}-\bar{X}\right)}{\sum_{i=1}^{n}\left(X_{i}-\bar{X}\right)^{2}}
$$

Então, a variância de $\bar{X}$ será estimada por (SEILA, 1992) (Equação 5):

$$
\hat{\sigma}_{\bar{X}}^{2}=\frac{\hat{\sigma}}{n}\left[1+2 \sum_{j=1}^{\infty} \hat{\rho}_{j}\right]
$$

Se as observações forem independentes, então $\rho_{j}=0$ para qualquer j e então $\sigma_{\bar{X}}^{2}=\sigma^{2} / n$. Como consequência, sendo $s^{2}$ o estimador para a variância de $X$, tem-se que $s^{2} / n$ é um estimador não viesado para $\sigma_{\bar{X}}^{2}$ (SElLA, 1992). No entanto, na presença de autocorrelação, o somatório introduz um viés na variância amostral da média, gerando intervalos de confiança mais estreitos do que os gerados a partir de dados não correlacionados. Nesta situação, $s^{2} / n$ passa a ser um estimador fortemente viesado para a variância (ALEXOPOULOS, 1994).

\section{Materiais e métodos}

Segundo Macke (2006), o método representa o conjunto de atividades sistemáticas e racionais orientando a geração de conhecimento válido, verdadeiro e indicando o caminho a ser seguido. 0 presente estudo foi realizado na forma de uma pesquisa quantitativa exploratória com características de estudo de caso (YIN, 2005). Conforme Macke (2006), essa abordagem é adequada para o estudo de subsistemas específicos nos quais o pesquisador atua como observador, descrição que se enquadra no propósito e forma de condução da presente pesquisa. Segundo Santos (1999), as pesquisas exploratórias têm como principal finalidade desenvolver, esclarecer e modificar conceitos e ideias, com vistas à formulação de problemas mais precisos ou hipóteses pesquisáveis para estudos posteriores. Segundo Eisenhardt (1989), os estudos de caso podem ser utilizados com a finalidade de descrição de um fenômeno ou para testar ou gerar teorias. Uma teoria, segundo essa autora, é uma afirmação a respeito da natureza da relação entre conceitos. Ela é gerada dentro de um contexto cognitivo próprio do pesquisador e reflete como ele percebe o ambiente de investigação.

0 objeto de estudo foi o nível de tensão de dois Circuitos de Distribuição (CD). Os dados foram analisados, seguindo os procedimentos da R505/2001 da ANEEL e, posteriormente, por meio de uma abordagem alternativa, fundamentada em conceitos de controle estatístico de processo. Os resultados foram comparados, visando identificar possíveis contribuições aos procedimentos de análise vigentes. A fim de permitir a comparação entre 0 processo definido pela Resolução n. ${ }^{0}$ 505/2001 da ANEEL e a abordagem estatística aqui proposta, o procedimento de análise consistiu nas seguintes etapas:

a) Coleta de dados: Os dados foram coletados durante o ano de 2007, pelo Departamento de Manutenção de Redes de uma concessionária de energia atuante no estado do Rio Grande do Sul, em dois circuitos de distribuição diferentes, ambos de baixa tensão (denominados CD01 e CD02). Ambos os circuitos foram classificados como adequados, do ponto de vista técnico dos recursos físicos utilizados;

b) Análise dos dados conforme o procedimento previsto na R505/2001 da ANEEL;

c) Análise da autocorrelação da série dos dados;

d) Eliminação da autocorrelação, se existente;

e) Análise da distribuição dos dados;

f) Cálculo dos índices de capacidade e capabilidade de processo; e

g) Compilação e comparação dos resultados.

Os dados foram analisados com auxílio do software estatístico Minitab versão 15, sendo utilizados procedimentos de cálculo dos índices de autocorrelação, capacidade e capabilidade. Os resultados obtidos são apresentados e discutidos nas seções seguintes.

\section{Análise do circuito de distribuição segundo Resolução n. ${ }^{0}$ 505/2001 da ANEEL}

Conforme o procedimento recomendado pela ANEEL, durante sete dias, foram coletadas 1.008 medidas no CD 01. Destas, 995 encontraram-se na faixa de tensão adequada, 10 na faixa precária inferior e 3 na faixa precária superior, conforme ilustrado na Figura 2. Os indicadores DRP e DRC foram calculados e resultaram nos índices de 1,3\% e 0,0\% respectivamente para o CD 01 .

Para estabelecer a classificação da tensão de atendimento do CD 01, primeiramente analisou-se o DRC. Como o índice DRC é 0,0\%, então o CD não é classificado como crítico e, logo, procedeu-se a análise do indicador DRP. Pelo critério estabelecido, caso o DRP seja maior que 3\%, o CD é rotulado como precário, do contrário entra como adequado. 
Com base nessas premissas, o CD 01 foi classificado como adequado.

Da mesma forma, para o CD 02 foram coletadas 1.008 medidas, todas obtidas na faixa de tensão adequada. Consequentemente, para o CD 02 os resultados foram $0,0 \%$ e $0,0 \%$ e o CD 02 foi considerado adequado. Os resultados são compilados na Tabela 1.

\section{Análise do circuito de distribuição pela abordagem estatística alternativa}

A proposta de análise dos CDs com utilização de técnicas estatísticas iniciou-se com a análise de autocorrelação dos dados coletados. Para fins de comparação, foram utilizados os mesmos dados das 1.008 medidas de tensão da análise anterior. 0 objetivo é garantir que os dados da amostra sejam independentes. Segundo o procedimento estabelecido pela ANEEL, os dados são coletados em intervalos sequenciais de 10 minutos, o que pode levar à autocorrelação entre as medidas. Para analisar essa possibilidade, procedeu-se à análise de autocorrelação dos dados. 0 nível de significância considerado foi de 5\%.

0 resultado para o CD 01 é apresentado na Figura 3. Conforme se pode perceber, há evidências de que existe autocorrelação dos dados do CD 01 com um lag associado à vigésima segunda amostra consecutiva. Isso equivale a considerar a existência de autocorrelação no espaço de tempo de 220 minutos entre as tensões coletadas.

A fim de eliminar a autocorrelação, foram selecionadas amostras sistemáticas dos dados coletados com intervalo de 230 minutos entre si. Cada amostra testada apresentou, consequentemente, 46 dados. 0 efeito de autocorrelação foi removido, não sendo mais considerado significante, conforme apresenta o exemplo de uma das amostras, ilustrado na Figura 4.

Com base nas amostras validadas quanto à autocorrelação, procedeu-se um teste de ajustamento de curvas, aplicando os testes de AndersonDarling e Kolmogorov-Smirnov (VACCARO, 1997), considerando-se os modelos exponencial, normal, Gamma e Weibull (NELSON, 1982). Outros modelos foram testados, tais como os modelos lognormal, logístico, loglogístico, de valores extremos superiores e de valores extremos inferiores. No entanto, os ajustes mais profícuos foram os apresentados pelos quatro modelos indicados. 0 objetivo foi identificar um modelo probabilístico para a distribuição de dados de tensão. 0 resultado dos testes para esse conjunto de dados indicou que o modelo de Weibull apresentou-se como promissor para representar a distribuição dos dados ( $p>0,25)$, não sendo encontradas evidências para rejeitá-lo com significância de 5\%.

0 próximo passo da abordagem proposta foi a análise dos índices de capacidade, a qual foi efetuada em duas instâncias. A primeira avaliou a capacidade para a classificação da tensão de atendimento crítica e a segunda para o nível precário.

0 índice de $\mathrm{P}_{\mathrm{pk}}$ para os limites de tensão críticos foi de 1,23 , não caracterizando, assim, o circuito de distribuição como crítico. A Figura 5

Classificação das tensões nominais - CD 01

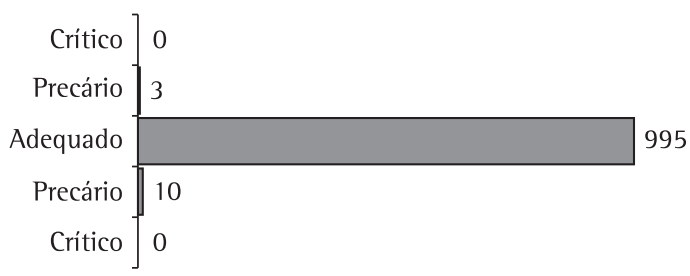

Figura 2. Classificação das tensões nominais - CD 01.

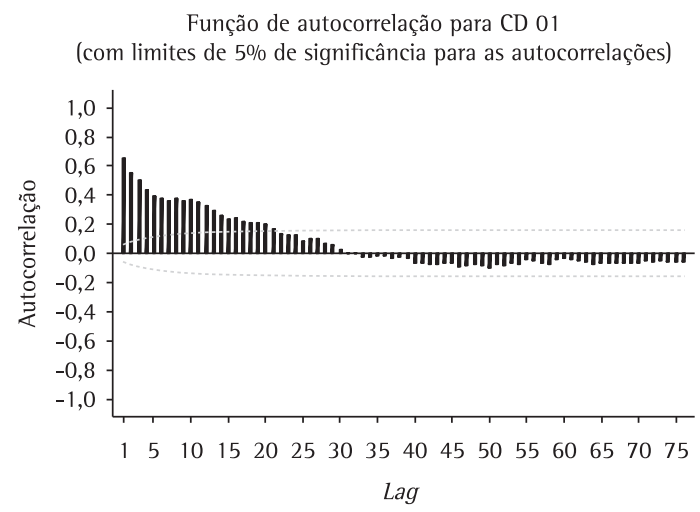

Figura 3. Autocorrelação de tensões do CD 01 com intervalos de coleta de 10 minutos, gerada com auxílio do Minitab 15 .

Tabela 1. Indicadores de medição dos CDs analisados.

\begin{tabular}{|c|c|c|c|c|c|c|c|c|}
\hline \multirow{2}{*}{ CD } & \multicolumn{5}{|c|}{ Medição por faixa } & \multicolumn{2}{|c|}{ Indicadores } & \multirow{2}{*}{ Classificação } \\
\hline & Crítico & Precário & Adequado & Precário & Crítico & DRP (\%) & DRC (\%) & \\
\hline 01 & 0 & 10 & 995 & 3 & 0 & 1,3 & 0,0 & Adequado \\
\hline 02 & 0 & 0 & 1008 & 0 & 0 & 0,0 & 0,0 & Adequado \\
\hline
\end{tabular}


apresenta outros resultados da análise realizada, inferindo-se que a probabilidade de gerar tensões na região de classificação crítica seria inferior a 0,03\%. No entanto, essa probabilidade representa, aproximadamente, uma ocorrência potencial a cada 18 meses (dado que as medições consideradas possuem intervalos de 230 minutos entre si).

Para os limites de tensão precária, o índice de $P_{p k}$ foi de 0,74 e o de $P_{p}$ igual a 0,90. Dessa forma, pode-se afirmar que esse circuito encontrar-se-ia, conforme o procedimento apresentado, na classificação precária. Além disso, como o $\mathrm{P}_{\mathrm{p}}$ tem o índice menor que 1 e diferente do $\mathrm{P}_{\mathrm{pk}}$, seriam necessárias ações para redução da variabilidade da tensão visando melhorar o índice $P_{p}$ e deslocar a média das tensões, com o objetivo de igualar o $P_{p k}$ e o $P_{p}$. A Figura 6 apresenta outros resultados da análise realizada, inferindo-se que a probabilidade de gerar tensões na classificação precária seria de

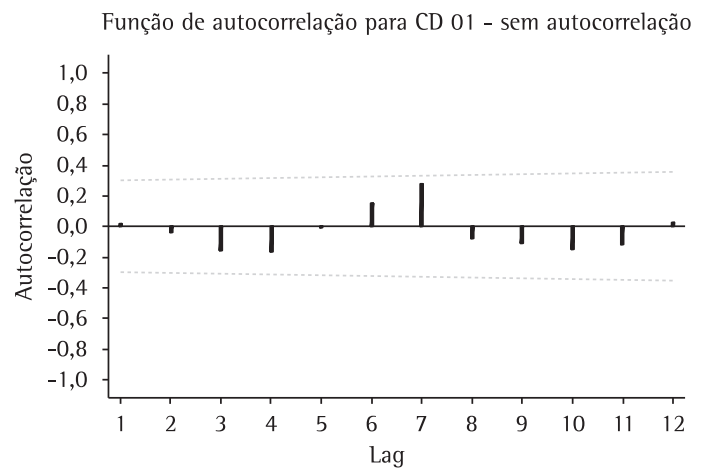

Figura 4. Autocorrelação de tensões CD 01 com intervalos de coleta de 230 minutos, gerada com auxílio do Minitab 15. cerca de $0,7 \%$, ou cerca de uma ocorrência a cada 23 dias.

0 mesmo método empregado na análise da classificação da tensão de atendimento do CD 01 foi utilizado no CD 02. A autocorrelação dos dados, com nível de significância igual a 5\%, foi identificada até a vigésima sexta coleta. 0 mesmo procedimento de tomar amostras sistemáticas, nesse caso com espaçamento temporal de 280 minutos, foi utilizado. As amostras foram analisadas quanto a sua distribuição e o modelo normal não pode ser rejeitado ( $p>0,29)$.

0 índice $P_{p k}$ para o nível crítico foi de 1,86, com $\mathrm{P}_{\mathrm{p}}$ de 4,78 (Figura 7). Para a classificação precária, o $\mathrm{P}_{\mathrm{pk}}$ mensurado foi de 0,90, com $\mathrm{P}_{\mathrm{p}}$ de 3,11 (Figura 8), representando uma ocorrência a cada dois meses, aproximadamente. Nesse caso, evidenciou-se que um ajuste na tensão média de atendimento levaria o CD 02 ao nível de classificação adequado.

\section{Análise e discussão}

0 uso da abordagem estatística proposta para a classificação da tensão de atendimento permitiu identificar resultados divergentes dos oriundos dos procedimentos descritos na resolução da ANEEL (BRASIL, 2008). Observa-se que o procedimento indicado pela resolução da ANEEL não exclui a possibilidade de ocorrência de tensões de atendimento fora da especificação nos casos analisados tampouco aponta quais os direcionamentos de melhoria necessários, indicando conformidade dos circuitos. No entanto,

Capabilidade (nível crítico) - CD 01 - sem autocorrelação

Baseado em Modelo Weibull

\begin{tabular}{|ll|}
\hline \multicolumn{2}{|c|}{ Dados de processo } \\
LSL & 109,22 \\
Meta & 127 \\
USL & 134,62 \\
Média amostral & 124,85 \\
N amostral & 46 \\
Forma & 56,354 \\
Escala & 126,176 \\
\hline \hline \multicolumn{2}{|c|}{ Desempenho observado } \\
PPM < LSL & 0,00 \\
PPM > USL & 0,00 \\
PPM Total & 0,00 \\
\hline
\end{tabular}

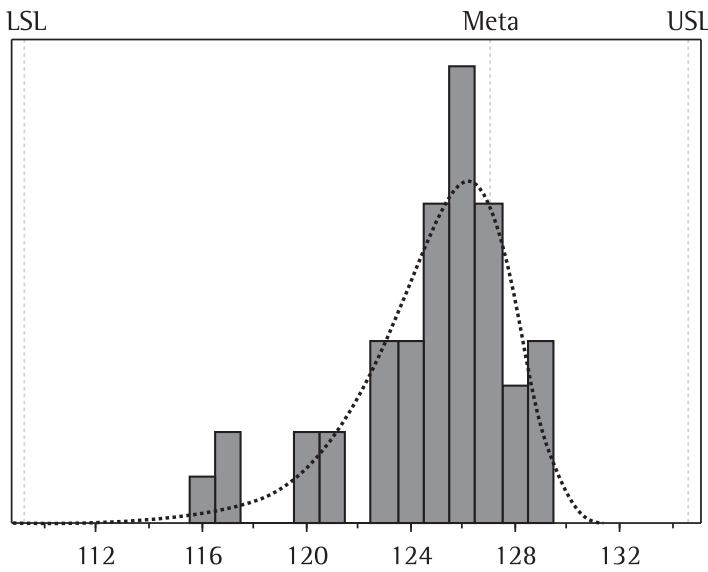

\begin{tabular}{|cc|}
\hline \multicolumn{3}{|c|}{ Capabilidade geral } \\
Pp & 1,39 \\
PPL & 1,23 \\
PPU & 1,81 \\
Ppk & 1,23 \\
\hline
\end{tabular}

Desempenho geral esperado

PPM $<$ LSL 293,79

PPM > USL $\quad 0,00$

PPM Total 293,79

112

Figura 5. Índice de capacidade para os limites de tensão no nível de classificação crítico - CD 01, gerada com auxílio do Minitab 15. 
Capabilidade (nível precário) - CD 01 - sem autocorrelação

Baseado em Modelo Weibull

\begin{tabular}{|ll|}
\hline \multicolumn{2}{|c|}{ Dados de processo } \\
LSL & 115,57 \\
Meta & 127 \\
USL & 132,08 \\
Média amostral & 124,85 \\
N amostral & 46 \\
Forma & 56,354 \\
Escala & 126,176 \\
\hline \hline Desempenho observado \\
PPM < LSL & 0,00 \\
PPM > USL & 0,00 \\
PPM Total & 0,00 \\
\hline
\end{tabular}

LSL

Meta USL

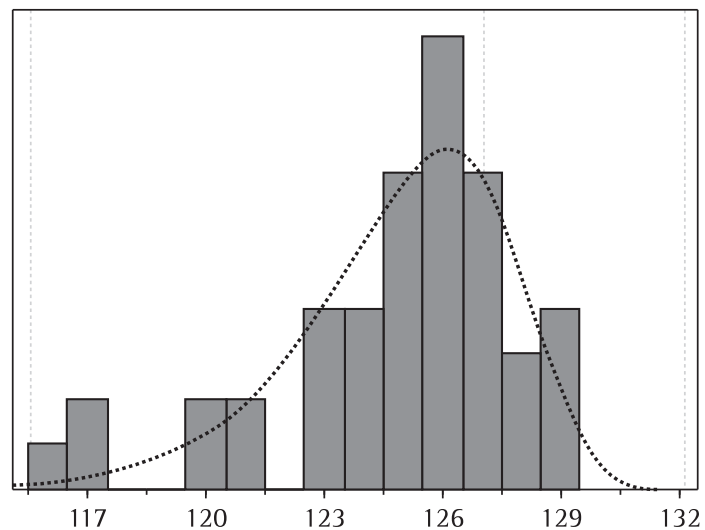

\begin{tabular}{|cc|}
\hline \multicolumn{3}{|c|}{ Capabilidade geral } \\
Pp & 0,90 \\
PPL & 0,74 \\
PPU & 1,31 \\
Ppk & 0,74 \\
\hline
\end{tabular}

Desempenho geral observado PPM < LSL 7073,80

PPM $>$ USL $\quad 1,93$

PPM Total 7075,73

Capabilidade (Nível Crítico) - CD 02 - Sem Autocorrelação

Figura 6. Índice de capacidade para os limites de tensão no nível de classificação precário - CD 01, gerada com auxílio do Minitab 15.

LSL

Meta

USL

\begin{tabular}{|ll|}
\hline \multicolumn{2}{|c|}{ Dados de processo } \\
LSL & 109,22 \\
Meta & 127 \\
USL & 134,62 \\
Média Amostral & 129,687 \\
N Amostral & 38 \\
dp (Potencial) & 0,809852 \\
dp (Geral) & 0,885063 \\
\hline
\end{tabular}

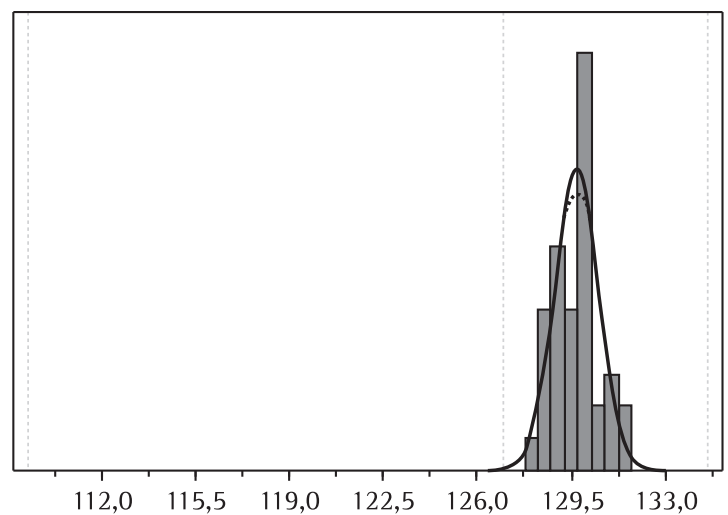

\begin{tabular}{|cc|}
\hline \multicolumn{3}{|c|}{ …..... Potencial } \\
\hline \multicolumn{2}{|c|}{ Geral } \\
\hline Cpabilidade & potencial \\
CPL & 5,23 \\
CPU & 8,42 \\
Cpk & 2,03 \\
Capabilidade geral & 2,03 \\
Pp & 4,78 \\
PPL & 7,71 \\
PPU & 1,86 \\
Ppk & 1,86 \\
Cpm & 0,89 \\
\hline
\end{tabular}

\begin{tabular}{|cc|}
\hline \multicolumn{2}{|c|}{ Desempenho observado } \\
PPM < LSL & 0,00 \\
PPM > USL & 0,00 \\
PPM Total & 0,00
\end{tabular}

\begin{tabular}{|cc|}
\hline Desempenho potencial esperado \\
PPM < LSL & 0,00 \\
PPM > USL & 0,00 \\
PPM Total & 0,00 \\
\hline
\end{tabular}

\begin{tabular}{|cc|}
\hline Desempenho geral esperado \\
PPM < LSL & 0,00 \\
PPM > USL & 0,01 \\
PPM Total & 0,01 \\
\hline
\end{tabular}

Figura 7. Índice de capacidade para os limites de tensão no nível de classificação crítico - CD 02, gerada com auxílio do Minitab 15.

essa possibilidade pode ser observada por meio da análise dos indicadores $P_{p k}$.

Um ponto central de discussão sobre a abordagem alternativa utilizada refere-se ao cuidado com a autocorrelação, dado que esta representa um pressuposto relevante sobre a aplicação de análises estatísticas sobre dados com coleta sistemática. Assim, a autocorrelação foi primeiramente detectada e eliminada. 0 intervalo de coleta utilizado na análise considerou, então, intervalos de tempo adequados para que os efeitos da autocorrelação não fossem significantes, caracterizando independência entre as coletas. Eliminados tais efeitos, modelos teóricos de distribuição podem ser testados.

No caso analisado foram testados diferentes modelos probabilísticos para compreender o padrão de distribuição dos dados coletados e verificar se o pressuposto de normalidade, hipótese de muitas abordagens estatísticas, poderia ser aceito. Uma vez ajustado um modelo teórico foram realizadas inferências sobre a população de dados e sua adequação aos parâmetros de qualidade de tensão estabelecidos pela ANEEL. 
média da ordem de 0,1 desvios padrão com poder de $90 \%$ e significância de 5\%. No entanto, dada a não independência entre as observações, esse poder é severamente comprometido. Além disso, a análise sugerida permite uma avaliação sobre o padrão de comportamento do circuito de distribuição e não somente de seu estado no período analisado. Nesse sentido, o uso de modelos probabilísticos e de testes de ajuste fornece uma alternativa para inferir sobre a população de tensões recebidas. Com isso torna-se possivel inferir sobre a tensão de atendimento do circuito de distribuição.

0 Quadro 2 apresenta um resumo comparativo entre as características do método estabelecido pela ANEEL e a abordagem proposta neste artigo.

\section{Conclusões}

0 presente estudo propõe uma abordagem, fundamentada em métodos probabilísticos de análise de séries de dados e de capacidade de processo, para a avaliação dos níveis de qualidade das tensões em circuitos de distribuição de energia elétrica. Essa abordagem é alternativa à sugerida pela ANEEL em sua Resolução n. ${ }^{\circ}$ 505/2001 no que concerne ao método de coleta e análise dos dados, mas respeita os padrões de qualidade por ela estabelecidos.

A análise foi realizada de forma comparativa, permitindo observar que os dados coletados sofrem forte influência de autocorrelação positiva, podendo gerar estimativas excessivamente otimistas quanto à variabilidade das tensões recebidas.

Ainda que evidências empíricas mostrem que a violação de níveis de tensão tende a ser persistente e está associada a causas especiais tais como defeitos em transformadores ou subsistemas, ou ainda sobrecarga na rede ou ações de manobra ou manutenção (BRITO, 2007; KREFTA, 2008), a questão sob análise avalia mais focadamente 0 impacto sobre a qualidade da informação gerada pelo procedimento de análise utilizado.

$\mathrm{Na}$ abordagem vigente, a análise dos dados relativos à conformidade da tensão de fornecimento considera tão somente os critérios de adequação estabelecidos pela ANEEL, os quais são fundamentados apenas na contagem das ocorrências em cada nível. Não há uma análise mais criteriosa dos dados sob o prisma estatístico, o que restringe a análise a um perfil descritivo e póstumo, perdendo-se a possibilidade de avaliação de padrões de comportamento e a inclusão de ações preditivas sobre a variabilidade e centralidade dos níveis de tensão relativamente aos parâmetros de qualidade estabelecidos pela ANEEL.
Uma abordagem estatística permitiria melhor conhecimento do comportamento dos níveis de tensão ao longo do tempo. Esse conhecimento pode ajudar a desenvolver alternativas técnicas de alterações nos sistemas elétricos de distribuição que visam melhorar a qualidade da tensão de fornecimento, com os menores custos possíveis. Além disso, permite qualificar o serviço prestado pelas concessionárias ao consumidor, revertendo-se em menos danos e custos associados a litígios e reparos, bem como na elaboração de planos de manobras que aproveitem a efetiva capacidade ociosa da rede, auxiliem na programação de desligamentos, objetivando a minimização das interrupções por falta de energia, na supervisão da qualidade do fornecimento, no controle de tensão em subestações, alimentadores e redes secundárias, e nos estudos de planejamento do sistema elétrico de distribuição.

\section{Referências}

AKAO, Y.; MIZUNO, S. QFD: the customer-driven approach to quality planning and deployment. Tokyo: Asian Productivity Organization, 1994. 356 p.

ALEXOPOULOS, C. A review of advanced methods for simulation output analysis. In: WINTER SIMULATION CONFERENCE, 1994, San Diego. Proceedings... Atlanta: IEEE, 1994. http://dx.doi.org/10.1109/WSC.2005.1574250

AMERICAN SOCIETY FOR QUALITY CONTROL - ASQC. Statistical process control manual. Milwaukee: ASQC, 1986.

BELISÁRIO, C. D. A.; BAHIENSE, D. A.; OLIVEIRA, G. M. Continuidade nos serviços de distribuição de energia elétrica. Conjuntura \& Planejamento, n. 105, p. 36-40, 2003.

BLANCHET, L. A. Concessão de serviços públicos. 2. ed. Curitiba Juruá, 1999. 323 p.

BRASIL. Presidência da República. Casa Civil. Lei No 8987 de 13 de fevereiro de 1995. Diário Oficial da República Federativa do Brasil, Brasília, DF, 14 fev. 1995. Disponível em: <http:// www.planalto.gov.br/ccivil_03/LEIS/L8987cons.htm>. Acesso em: 15/07/2009.

BRASIL. Agência Nacional de Energia Elétrica - ANEEL. Atlas de Energia Elétrica do Brasil. Disponivel em: <http://www. aneel.gov.br/arquivos/PDF/atlas_par1_cap2.pdf>. Acesso em: 30 jul. 2009.

BRASIL. Agência Nacional de Energia Elétrica - ANEEL. Procedimentos de Distribuição de Energia Elétrica no Sistema Elétrico Nacional - PRODIST. Disponível em: <http://www. aneel.gov.br/arquivos/PDF/Modulo8_24032006_SRD.pdf>. Acesso em: 20 out. 2008.

BRASIL. Agência Nacional de Energia Elétrica - ANEEL. Resolução $n^{\circ}$ 505, de 26 de novembro de 2001. Diário Oficial da República Federativa do Brasil, Brasília, DF, 27 nov. 2001. v. 138, n. 226, Seção 1, p. 16. Disponível em: <http://www.aneel.gov.br/cedoc/res2001505.pdf>. Acesso em: 20 out. 2008.

BRITO, M. P. Geração distribuída: critérios e impactos na rede elétrica. 2007. 55 f. Trabalho de Conclusão (Engenharia Elétrica)-Universidade Federal do Espírito Santo, 2007.

CLARO, F. A. E.; COSTA, A. F. B.; MACHADO, M. A. G. Gráficos de controle de EWMA e de X-barra para monitoramento de processos autocorrelacionados. Produção, v. 17, n. 3 , 
p. 536-546, 2007. http://dx.doi.org/10.1590/S010365132007000300010

DEMING, W. E. Out of the crisis. Cambridge: MIT Press, 2000. $507 \mathrm{p}$.

DEUS, A. D.; VACCARO, G. L. R. Uma abordagem de implementação da qualidade assegurada no fornecimento de componentes automotivos: estudo de caso. In: ENCONTRO NACIONAL DE ENGENHARIA DE PRODUÇ̃̃O - ENEGEP, 28., 2008, Rio de Janeiro. Anais... Rio de Janeiro: ABEPRO, 2008.

EISENHARDT, K. M. Building theories from case study research. Academy of Management Review, v. 14, n. 4, p. 532-550, 1989.

FEIGENBAUM, A. V. Total quality control. 3. ed. New York: McGraw-Hill, 1983. 768 p.

GIL, A. C. Projetos de pesquisa. 3. ed. São Paulo: Atlas, 1996. 175 p.

GOLDEMBERG, J. Energia, meio ambiente \& desenvolvimento. São Paulo: Edusp; Cesp, 1998. 234 p.

GONÇALEZ, P. U.; WERNER, L. Comparação dos índices de capacidade do processo para distribuições não-normais. Gestão \& Produção, v. 16, n. 1, p. 121-132, 2009.

ITO, H. T. Energia elétrica: apuração da qualidade dos dados de consumo. 2003. 110 f. Dissertação (Mestrado em Engenharia Mecânica)-Universidade Estadual de Campinas, 2003.

JANNUZI, A. C. Regulação da qualidade de energia elétrica sob o foco do consumidor. 2007. 216 f. Dissertação (Mestrado em Engenharia Elétrica)-Universidade de Brasília, 2007.

JURAN, J. M. A qualidade desde o projeto: os novos passos para o planejamento da qualidade em produtos e serviços. São Paulo: Pioneira, 1992. 551 p.

KAMINSKY, F. C.; DOVICH, R. A.; BURKE, R. J. Process capability indices: now and in the future. Quality Engineering, v. 10, n. 3, p. 445-453, 1998. http://dx.doi. org/10.1080/08982119808919158

KREFTA, G. F. Avaliação da inclusão de unidades de medição sincrofasorial na estimação de estados de sistemas elétricos de potência. 2008. 186 f. Trabalho de Conclusão (Engenharia Elétrica)-Universidade Federal do Paraná, 2008.

LIN, Y. C.; CHOU, C. Y. On the design of variable sample size and sampling intervals $\bar{X}$ charts under non-normality. International Journal of Production Economics, n. 96, p. 249-261, 2004.

MACKE, J. A pesquisa-ação como estratégia de pesquisa qualitativa. In: GODOI, C.; BANDEIRA-DE-MELO, R.;
SILVA, A. Pesquisa qualitativa em estudos organizacionais: paradigmas, estratégias e métodos. São Paulo: Saraiva, 2006. p. 207-239.

MÉFFE, A. et al. Desenvolvimento de modelos e ferramenta computacional para avaliação dos riscos e minimização das violações de tensão em sistemas de distribuição. Manual Técnico da ENERSUL, 2002.

MONTGOMERY, D. C. Introdução ao controle estatístico da qualidade. 4. ed. Rio de Janeiro: LTC, 2004. 513 p.

MONTGOMERY, D. C.; RUNGER, G. C. Estatística aplicada e probabilidade para engenheiros. 2. ed. Rio de Janeiro: LTC, 2003. $463 \mathrm{p}$.

NELSON, W. Applied life data analysis. 5. ed. New York: John Wiley \& Sons, 1982. 570 p. http://dx.doi. org/10.1002/0471725234

PANIAGO, E. L. Dispositivo para monitoramento, registro e análise dos indicadores de continuidade no fornecimento da energia elétrica. 2006. 110 f. Dissertação (Mestrado em Engenharia Elétrica)-Universidade de Brasília, 2006.

SANTOS, A. R. Metodologia científica: a construção do conhecimento. 2. ed. Rio de Janeiro: DP\&A, 1999.

SCHAEFFER, R. et al. Energia e pobreza: problemas de desenvolvimento energético e grupos sociais marginais em áreas rurais e urbanas do Brasil. Santiago: CEPAL, 2003. 84 p.

SEILA, A. F. Advanced output analysis for simulation. In: WINTER SIMULATION CONFERENCE, 1992, San Diego. Proceedings... Atlanta: IEEE, 1992.

SHEWHART, W. A. Economic Control of Quality of Manufactured Product. New York: Van Nostrand, 1931. 501 p.

THESEN, A.; TRAVIS, L. E. Introduction to Simulation. In: WINTER SIMULATION CONFERENCE, 1994, San Diego. Proceedings... Atlanta: IEEE, 1990.

TORMINATO, S. M. Análise da utilização da ferramenta CEP: um estudo de caso na manufatura de autopeças. Dissertação (Mestrado em Engenharia Mecânica)- Universidade Estadual de Campinas, 2004, 106 p.

VACCARO, G. L. R. Modelagem e análise da confiabilidade de sistemas. 1997. 222 f. Dissertação (Mestrado em Engenharia de Produção)-Universidade Federal do Rio Grande do Sul, 1997.

YIN, R. K. Estudo de caso: planejamento e métodos. Porto Alegre: Bookman, 2005. 211 p.

\title{
Statistical analysis of the quality of tension levels in electrical energy distribution systems
}

\begin{abstract}
This paper proposes a statistical approach to analyze the quality of voltage levels in electric Power distribution systems, based on the principles established by ANEEL Resolution 505/2001, under which all utility power distribution of the country are subjected. The paper presents considerations on quality in electricity distribution, existing procedures for quality control of voltage levels and suggests the possibility of using statistical process control concepts and levels of ability and performance as evidence of alternative information and prediction. A case study in two circuits of low voltage distribution systems is analyzed comparatively, using primarily the procedures and criteria established by ANEEL, and then the approach proposed by the authors. The article concludes by analyzing the potential contributions of the statistical procedures proposed.
\end{abstract}

\section{Keywords}

Electric energy distribution. Tension level control. Quality. Statistical process control. Autocorrelation. 